\title{
Spectral extremal results for hypergraphs
}

\author{
Yuan Hou \\ Department of Computer Engineering \\ Zhicheng College \\ Fuzhou University \\ Fuzhou, Fujian, P.R. China \\ houyuan@fzu.edu.cn \\ An Chang*
}

Center for Discrete Mathematics and Theoretical Computer Science

Fuzhou University

Fuzhou, Fujian, P.R. China

anchang@fzu.edu.cn

Joshua Cooper

Department of Mathematics

University of South Carolina

Columbia, SC, U.S.A.

cooper@math.sc.edu

Submitted: Sep 17, 2019; Accepted: Aug 6, 2021; Published: Aug 27, 2021

(C) The authors. Released under the CC BY-ND license (International 4.0).

\begin{abstract}
Let $F$ be a graph. A hypergraph is called Berge $F$ if it can be obtained by replacing each edge in $F$ by a hyperedge containing it. Given a family of graphs $\mathcal{F}$, we say that a hypergraph $H$ is Berge $\mathcal{F}$-free if for every $F \in \mathcal{F}$, the hypergraph $H$ does not contain a Berge $F$ as a subhypergraph. In this paper we investigate on the connections between spectral radius of the adjacency tensor and structural properties of a linear hypergraph. In particular, we obtain a spectral version of Turán-type problems over linear $k$-uniform hypergraphs by using spectral methods.

Mathematics Subject Classifications: 05C50, 05C65, 05C35
\end{abstract}

*Supported by the National Natural Science Foundation of China. 


\section{Introduction}

A hypergraph $H=(V, E)$ consists of a vertex set $V$ and an edge (hyperedge) set $E$, where each edge is a nonempty subset of $V$. A hypergraph is called $k$-uniform if each edge is a $k$-element subset of $V$. A 2-uniform hypergraph is simply called a graph. Two vertices $x$ and $y$ are said to be adjacent if there is an edge that contains both of these vertices. A hypergraph $H$ is called linear if every two edges have at most one vertex in common.

For a fixed $k$-uniform family $\mathcal{F}$, the Turán number of $\mathcal{F}$, denoted by $e x_{k}(n, \mathcal{F})$, is the maximum number of edges of an $\mathcal{F}$-free hypergraph on $n$ vertices. Similarly, given a family of $k$-uniform linear hypergraphs $\mathcal{F}$, the linear Turán number of $\mathcal{F}$, denoted $\operatorname{ex}_{k}^{\operatorname{lin}}(n, \mathcal{F})$, is the maximum number of edges in an $\mathcal{F}$-free $k$-uniform linear hypergraph on $n$ vertices.

Turán type extremal problems in graphs and hypergraphs are the central topic of extremal combinatorics and have a vast literature. For a survey of recent results we refer the reader to $[11,18,22]$. Only a handful of results are known about the asymptotic behaviour of Turán numbers for hypergraphs. One of the most active subjects is Berge hypergraphs. The classical definition of a hypergraph cycle due to Berge is the following: a Berge cycle $C_{t}$ of length $t \geqslant 2$ is an alternating sequence of distinct vertices (other than first and last) and distinct edges of the form $v_{1} l_{1} v_{2} l_{2} \cdots v_{t} l_{t}$ where $v_{i}, v_{i+1} \in l_{i}$ for each $i \in\{1,2, \cdots, t-1\}$ and $v_{t}, v_{1} \in l_{t}$.

Gerbner and Palmer [14] gave the following natural generalization of the definitions of Berge graphs. Let $F=(V(F), E(F))$ be a graph and $\mathcal{B}=(V(\mathcal{B}), E(\mathcal{B}))$ be a hypergraph. We say $\mathcal{B}$ is Berge $F$ if there is a bijection $\phi: E(F) \rightarrow E(\mathcal{B})$ such that $e \subseteq \phi(e)$ for all $e \in E(F)$. In other words, given a graph $F$, we can obtain a Berge $F$ by replacing each edge of $F$ with a hyperedge that contains it. Given a family of graphs $\mathcal{F}$, we say that a hypergraph $H$ is Berge $\mathcal{F}$-free if for every $F \in \mathcal{F}$, the hypergraph $H$ does not contain a Berge $F$ as a subhypergraph. The maximum possible number of edges in a Berge $\mathcal{F}$-free hypergraph on $n$ vertices is the Turán number of Berge $\mathcal{F}$.

Linear Turán extremal problems have been studied mostly implicitly. For example, the famous upper bound of Ruzsa and Szemerédi [26] on triple systems not carrying three edges on six vertices is equivalent to $e x_{3}^{\operatorname{lin}}(n, T)=o\left(n^{2}\right)$ where $T$ is the linear triangle. For $l \geqslant 2$, Füredi and Özkahya [10] showed $e x_{3}^{\operatorname{lin}}\left(n, C_{2 l+1}\right) \leqslant 2 \ln n^{1+1 / l}+9 \ln$. For the even case, it is easy to show $e x_{3}^{\operatorname{lin}}\left(n, C_{2 l}\right) \leqslant O\left(n^{1+1 / l}\right)$. Recently, linear Turán numbers were studied in $[5,8,12,15,28]$.

Our aim is to consider a spectral version of hypergraph Turán problems, i.e., spectral extremal hypergraph theory, which is the subset of extremal problems where invariants are based on the eigenvalues or eigenvectors of a hypergraph. The most natural such invariant is the maximal absolute value of the eigenvalues of the adjacency tensor of a hypergraph $H$, called its spectral radius. Because the spectral radius is a close correlate of the number of edges in a hypergraph, the following problem is a natural spectral analog of hypergraph Turán problems: What is the maximum spectral radius of hypergraphs of order $n$, not containing a given $\mathcal{F}$ ?

In fact, spectral extremal graph theory has a substantial history, with many important results. Examples include Stanley's bound [27], theorems of Wilf [29] relating eigenvalues 
of graphs to their chromatic number, and many other examples. Much of recent work in spectral extremal graph theory is due to Nikiforov, who has considered maximizing the spectral radius over several families of graphs; see [23]. Although the area is still difficult and underdeveloped, we believe that ultimately a spectral approach to extremal hypergraph theory will turn out to be a fruitful and interesting accompaniment to "conventional" extremal theory, see e.g., $[1,17]$.

The rest of this paper is organized as follows. In the next section, the bulk of the necessary notation and the basic facts have been presented, including the definitions and properties of eigenvalues of tensors and hypergraphs. We develop a few new tools in order to provide spectral analogues of extremal hypergraph problems. In Section 3 we consider spectral analogues of Turán-type problems for hypergraphs and investigate bounds on the maximum spectral radius of linear $k$-uniform hypergraphs with girth at least five by using the spectral methods proved in Section 2.

\section{Preliminaries}

\section{$2.1 \quad$ Spectra of tensors}

In 2005, Qi [25] and Lim [20] independently introduced the concept of tensor eigenvalues and the spectra of tensors. An order $k$ dimension $n$ real tensor ${ }^{1} \mathcal{T}=\left(\mathcal{T}_{i_{1} \cdots i_{k}}\right)$ consists of $n^{k}$ real entries $\mathcal{T}_{i_{1} \cdots i_{k}}$ for $1 \leqslant i_{1}, i_{2}, \cdots, i_{k} \leqslant n$. Evidently, a vector of dimension $n$ is a tensor of order 1 and a matrix is a tensor of order 2. $\mathcal{T}$ is called symmetric if the value of $\mathcal{T}_{i_{1} \cdots i_{k}}$ is invariant under any permutation of the indices $i_{1}, i_{2}, \cdots, i_{k}$. Given a vector $x \in \mathbb{R}^{n}, \mathcal{T} x^{k}$ is a real number and $\mathcal{T} x^{k-1}$ is an $n$-dimensional vector. $\mathcal{T} x^{k}$ and the $i$ th component of $\mathcal{T} x^{k-1}$ are given by:

$$
\begin{aligned}
\mathcal{T} x^{k} & =\sum_{i_{1}, i_{2}, \cdots, i_{k} \in[n]} \mathcal{T}_{i_{1} i_{2} \cdots i_{k}} x_{i_{1}} x_{i_{2}} \cdots x_{i_{k}} . \\
\left(\mathcal{T} x^{k-1}\right)_{i} & =\sum_{i_{2}, \cdots, i_{k} \in[n]} \mathcal{T}_{i i_{2} \cdots i_{k}} x_{i_{2}} \cdots x_{i_{k}} .
\end{aligned}
$$

The former is simply tensor contraction of $\mathcal{T}$ with the $k$-th outer product of $x$ with itself, and the latter is the $i$-th coordinate of the contraction of $\mathcal{T}$ with the $(k-1)$-st outer product of $x$ with itself. Note that the symmetry of $\mathcal{T}$ makes these contractions well-defined without specifying which indices are summed over. Let $\mathcal{T}$ be an order $k$ dimension $n$ real tensor. For some $\lambda \in \mathbb{C}$, if there exists a nonzero vector $x \in \mathbb{C}^{n}$ satisfying the eigenequation

$$
\mathcal{T} x^{k-1}=\lambda x^{[k-1]},
$$

then $\lambda$ is a called an eigenvalue of $\mathcal{T}$ and $x$ is its corresponding eigenvector, where $x^{[k-1]}:=$ $\left(x_{1}^{k-1}, x_{2}^{k-1}, \cdots, x_{n}^{k-1}\right)^{T} \in \mathbb{C}^{n} \backslash\{0\}$.

If $x$ is a real eigenvector of $\mathcal{T}$, then clearly the corresponding eigenvalue $\lambda$ is real. In this case, $\lambda$ is called an $H$-eigenvalue and $x$ is called an $H$-eigenvector associated with

\footnotetext{
${ }^{1}$ Sometimes known as a "hypermatrix" or simply, "matrix."
} 
$\lambda$. Furthermore, if $x$ is nonnegative and real, we say $\lambda$ is an $H^{+}$-eigenvalue of $\mathcal{T}$. If $x$ is positive and real, $\lambda$ is said to be an $H^{++}$-eigenvalue of $\mathcal{T}$. The maximal absolute value of the eigenvalues of $\mathcal{T}$ is called the spectral radius of $\mathcal{T}$, denoted by $\rho(\mathcal{T})$.

In 2012, Cooper and Dutle [6] defined the adjacency tensor of a $k$-uniform hypergraph $H$. The adjacency tensor $\mathcal{A}=\mathcal{A}(H)$ is an order $k$ dimension $n$ symmetric tensor defined by

$$
\mathcal{A}_{i_{1} \cdots i_{k}}= \begin{cases}\frac{1}{(k-1) !} & \text { if }\left\{i_{1}, \cdots, i_{k}\right\} \in E \\ 0 & \text { otherwise. }\end{cases}
$$

This definition generalizes adjacency matrices, and its theory is a natural starting point for spectral hypergraph theory. For a vector $x$ of dimension $n$ and a subset $U \subseteq V$, we write

$$
x^{U}=\prod_{v_{i} \in U} x_{i} .
$$

The product $\mathcal{A} x^{k}$ therefore has an interpretation as follows:

$$
\mathcal{A} x^{k}=k \sum_{e \in E(H)} x^{e}
$$

The right-hand side (without the factor of $k$ ) is sometimes known as the Lagrangian polynomial of $H$.

For nonnegative tensors, we have a generalization of the Perron-Frobenius theorem, see $[4,6,13,30]$. Let $\mathcal{T}=\left(\mathcal{T}_{i_{1} \cdots i_{k}}\right)$ be an order $k$ dimension $n$ nonnegative tensor. If for any nonempty proper index subset $\alpha \subset\{1,2, \cdots, n\}$, there is at least an entry $\mathcal{T}_{i_{1} \cdots i_{k}}>0$, where $i_{1} \in \alpha$ and at least an $i_{j} \notin \alpha$ for $j=2, \cdots, k$, then $\mathcal{T}$ is called nonnegative weakly irreducible tensor. It was proved that a $k$-uniform hypergraph $H$ is connected if and only if its adjacency tensor $\mathcal{A}(H)$ is weakly irreducible (see $[13,30]$ ). Let $\rho(H)$ denote the spectral radius of a hypergraph $H$. By the Perron-Frobenius theorem, if $H$ is connected, the eigenvector $x=\left(x_{1}, x_{2}, \cdots, x_{n}\right)^{T}$ corresponding to $\rho(H)$, known as the principal eigenvector, can be chosen to be strictly positive. Throughout the paper, we only consider connected and simple hypergraphs.

\subsection{Useful tools}

In this section we present some useful tools which help to recast into spectral theory some classical results on hypergraphs and their proofs. We also introduce some additional notation employed below.

Let $H=(V, E)$ be a connected simple hypergraph on $n$ vertices and $m$ edges. For a vertex $v$, let $L(v)$ be the set of edges containing $v$ and $N_{v}$ be the neighborhood of $v$, i.e., $N_{v}=\{x \in V \backslash\{v\} \mid v, x \in l$ for some $l \in E\}$. The degree of a vertex $v$, which is denoted by $d(v)$, is defined as the number of edges containing $v$, i.e., $d(v)=|L(v)|$. For any two vertices $u$ and $v$, let $N_{u v}$ be the set of common neighbors of $u$ and $v$. The codegree of $u$ and $v$, denoted by $d(u, v)$, is the number of edges containing both $u$ and $v$ in $H$. Let $\Delta$, 
$\Delta_{2}$ and $\bar{d}$ denote the maximum degree, the maximum codegree and the average degree of $H$, respectively. It is easy to verify that $\bar{d}=k m / n$.

For a set $X \subseteq V$, let $E_{t}(X)=\{l \mid l \in E$ and $|l \cap X|=t\}$ and $e_{t}(X)=\left|E_{t}(X)\right|$. Similarly, let $E_{t}^{v}(X)=\{l \mid l \in E, v \in l$ and $|l \cap X|=t\}$ and $e_{t}^{v}(X)=\left|E_{t}^{v}(X)\right|$. If $X$ and $Y$ are disjoint sets of vertices of $H$, we write $E(X, Y)$ as the set of edges containing some vertices in $X$ and the others in $Y$ and $e(X, Y)$ the number of edges in $E(X, Y)$, i.e., $E(X, Y)=\{e \in E(H) \mid H \cap X \neq \varnothing$ and $H \cap Y \neq \varnothing\}$ and $e(X, Y)=|E(X, Y)|$.

Lemma 1. Let $H$ be a connected simple $k$-uniform hypergraph and $\rho$ be the spectral radius of the adjacency tensor of $H$. Then

$$
\rho^{2} \leqslant \frac{1}{k-1} \sum_{t=1}^{k} \sum_{e \in E_{t}\left(N_{u}\right)} \sum_{v \in N_{u} \cap e} d(u, v)
$$

where $u$ is the vertex corresponding to a maximum entry of the principal eigenvector.

Proof. Let $x$ be an eigenvector corresponding to $\rho$. For a vertex $v \in V(H)$, we will use $x_{v}$ to denote the eigenvector entry of $x$ corresponding to $v$. For any $v \in V(H)$, the eigenvector equation is

$$
\rho x_{v}^{k-1}=\sum_{\left\{v, i_{2}, \cdots, i_{k}\right\} \in E} x_{i_{2}} x_{i_{3}} \cdots x_{i_{k}} .
$$

By the Perron-Frobenius Theorem, $x$ has all positive entries, and it will be convenient for us to normalize so that the maximum entry of $x$ is 1 . Let $u$ denote the vertex with maximum eigenvector entry equal to 1 . Throughout the paper, we will use $u$ to denote the vertex with maximum eigenvector entry equal to 1 . If there are multiple such vertices, choose and fix $u$ arbitrarily among them. Since $x_{u}=1$, (6) becomes

$$
\rho=\sum_{\left\{u, i_{2}, \cdots, i_{k}\right\} \in E} x_{i_{2}} x_{i_{3}} \cdots x_{i_{k}} .
$$

Apply AM-GM inequality to (7), we have

$$
\rho \leqslant \frac{1}{k-1} \sum_{\left\{u, i_{2}, \cdots, i_{k}\right\} \in E}\left(x_{i_{2}}^{k-1}+x_{i_{3}}^{k-1}+\cdots+x_{i_{k}}^{k-1}\right) .
$$

The next inequality is a simple consequence of our normalization and an easy double counting argument, but will be used extensively throughout the paper and therefore warrants special attention. Multiplying both sides of (8) by $\rho$ and applying (6) gives

$$
\begin{aligned}
\rho^{2} & \leqslant \frac{1}{k-1} \sum_{\left\{u, i_{2}, \cdots, i_{k}\right\} \in E} \rho x_{i_{2}}^{k-1}+\rho x_{i_{3}}^{k-1}+\cdots+\rho x_{i_{k}}^{k-1} \\
& =\frac{1}{k-1} \sum_{\left\{u, i_{2}, \cdots, i_{k}\right\} \in E}\left[\sum_{e \in L\left(i_{2}\right)} x^{e \backslash\left\{i_{2}\right\}}+\sum_{e \in L\left(i_{3}\right)} x^{e \backslash\left\{i_{3}\right\}}+\cdots+\sum_{e \in L\left(i_{k}\right)} x^{e \backslash\left\{i_{k}\right\}}\right] .
\end{aligned}
$$


Since the maximum entry of $x$ is normalized to 1 , it is obvious that $x^{e \backslash\{v\}} \leqslant 1$ for any vertex $v \in V(H)$. Thus

$$
\rho^{2} \leqslant \frac{1}{k-1} \sum_{\left\{u, i_{2}, \cdots, i_{k}\right\} \in E}\left[\sum_{e \in L\left(i_{2}\right)} 1+\sum_{e \in L\left(i_{3}\right)} 1+\cdots+\sum_{e \in L\left(i_{k}\right)} 1\right] .
$$

We now estimate the right side of (9) in two different ways. On one hand, for a fixed edge $\left\{u, i_{2}, \cdots, i_{k}\right\} \in E$, we consider the edge set $L\left(i_{j}\right)$ for $2 \leqslant j \leqslant k$. The edge set $L\left(i_{j}\right)$ can be considered the union of the $k$ disjoint subsets of edges incident to $i_{j}$ which have intersection with $N_{u}$ of cardinality $t=1$ through $t=k$. That is, $L\left(i_{j}\right)=\bigcup_{t=1}^{k} E_{t}^{i_{j}}\left(N_{u}\right)$. Then we have $\sum_{e \in L\left(i_{j}\right)} 1=\sum_{t=1}^{k}\left(\sum_{e \in E_{t}^{i_{j}}\left(N_{u}\right)} 1\right)$. Combining with (9), this gives

$$
\rho^{2} \leqslant \frac{1}{k-1} \sum_{\left\{u, i_{2}, \cdots, i_{k}\right\} \in E}\left[\sum_{t=1}^{k}\left(\sum_{e \in E_{t}^{i_{2}}\left(N_{u}\right)} 1+\sum_{e \in E_{t}^{i_{3}}\left(N_{u}\right)} 1+\cdots+\sum_{e \in E_{t}^{i_{k}}\left(N_{u}\right)} 1\right)\right]
$$

On the other hand, for an arbitrary edge $l=\left\{i_{1}, i_{2}, \cdots, i_{k}\right\}$ with $\left|l \cap N_{u}\right|=t$, without loss of generality, assume $l \cap N_{u}=\left\{i_{1}, i_{2}, \cdots, i_{t}\right\}$. Then the contribution of the edge $l$ on the right-hand side of (10) is just the sum of the number of edges containing both the vertices $u$ and $i_{j}$ over $1 \leqslant j \leqslant t$. In other words, the edge $l$ appears in the right summation exactly $d\left(u, i_{1}\right)+d\left(u, i_{2}\right)+\cdots+d\left(u, i_{t}\right)$ times.

Thus (10) becomes

$$
\begin{aligned}
\rho^{2} & \leqslant \frac{1}{k-1}\left[\sum_{e \in E_{1}\left(N_{u}\right)} \sum_{v \in N_{u} \cap e} d(u, v)+\cdots+\sum_{e \in E_{k}\left(N_{u}\right)} \sum_{v \in N_{u} \cap e} d(u, v)\right] \\
& =\frac{1}{k-1} \sum_{t=1}^{k} \sum_{e \in E_{t}\left(N_{u}\right)} \sum_{v \in N_{u} \cap e} d(u, v) .
\end{aligned}
$$

This completes the proof.

Note that Lemma 1 illustrates a relationship between spectral radius of the adjacency tensor and structural properties of hypergraphs. Combined with estimates of the codegree, one can obtain results such as the following.

Lemma 2. Let $H$ be a connected simple $k$-uniform hypergraph with maximum codegree $\Delta_{2}$ and $\rho$ be the spectral radius of the adjacency tensor of $H$. Let $u$ be the vertex with maximum eigenvector entry. Then

(1) $\rho^{2} \leqslant \frac{\Delta_{2}}{k-1}\left[e_{1}\left(N_{u}\right)+2 e_{2}\left(N_{u}\right)+\cdots+k e_{k}\left(N_{u}\right)\right]$;

(2) $\rho^{2} \leqslant \frac{\Delta_{2}}{k-1} \sum_{v \in N_{u}} d(v)$. 
Proof. Since the maximum codegree of $H$ is $\Delta_{2}$, by Lemma 1, we have

$$
\rho^{2} \leqslant \frac{\Delta_{2}}{k-1}\left[e_{1}\left(N_{u}\right)+2 e_{2}\left(N_{u}\right)+\cdots+k e_{k}\left(N_{u}\right)\right]=\frac{\Delta_{2}}{k-1} \sum_{v \in N_{u}} d(v)
$$

where the last equality follows because both sums count the number of pairs $(v, e)$ where $v \in e \in \bigcup_{t} E_{t}\left(N_{u}\right)$.

It is clear that the codegree of each pair of adjacent vertices in $H$ is exactly 1 if $H$ is a linear hypergraph. We get the following result.

Corollary 3. Let $H$ be a connected simple $k$-uniform linear hypergraph and $\rho$ be the spectral radius of the adjacency tensor of $H$. Let $u$ be the vertex with maximum eigenvector entry. Then

(1) $\rho^{2} \leqslant \frac{1}{k-1}\left[e_{1}\left(N_{u}\right)+2 e_{2}\left(N_{u}\right)+\cdots+k e_{k}\left(N_{u}\right)\right]$;

(2) $\rho^{2} \leqslant \frac{1}{k-1} \sum_{v \in N_{u}} d(v)$.

For linear hypergraphs, the results in Corollary 3 are our main tools and we will use this technique in the sequel.

\section{Main Results}

The purpose of this section is to illustrate the use of the tools developed in Section 2, which translate nonspectral extremal problems into spectral results. We also determine bounds on the maximum spectral radius of uniform hypergraphs with girth at least five.

\subsection{Spectral radius of linear hypergraphs without Fan $^{k}$}

To illustrate this technique, we first give a spectral version result corresponding to the linear Turán number of $\mathrm{Fan}^{k}$. We include this as a quick way for the reader to become acquainted with our notation.

For $k \geqslant 2$, the $k$-fan $\mathrm{Fan}^{k}$ is the $k$-uniform linear hypergraph with $k$ edges $f_{1}, \cdots, f_{k}$ which pairwise intersect in the a single vertex $v$, and an additional edge $g$ which intersects all $f_{i}$ in a vertex different from $v$. Füredi and Gyárfás studied the linear Turán number of $\mathrm{Fan}^{k}$ in [12]. They proved that $e x_{k}^{\operatorname{lin}}\left(n, \operatorname{Fan}^{k}\right) \leqslant \frac{n^{2}}{k^{2}}$, asymptotically a factor of $1+1 /(k-1)$ smaller than the maximum number of edges in a $k$-uniform linear hypergraph, $\left(\begin{array}{l}n \\ 2\end{array}\right) /\left(\begin{array}{l}k \\ 2\end{array}\right)$. The Turán number of $\mathrm{Fan}^{k}$ on $k$-uniform hypergraphs was determined by Mubayi and Pikhurko in [21].

Theorem $4([12])$. One has $\operatorname{ex}_{k}^{\text {lin }}\left(n, \mathrm{Fan}^{k}\right) \leqslant \frac{n^{2}}{k^{2}}$ for all $k \geqslant 2$. The only extremal hypergraphs are the transversal designs on $n$ vertices with $k$ groups. 
A transversal design $T(n, k)$ on $n$ vertices with $k$ groups is a $k$-partite hypergraph with groups of equal size (thus $n$ is a multiple of $k$ ) and each pair of vertices from different groups is contained in exactly one hyperedge. Such designs have long been known to exist for all sufficiently large $n$ (as a function of $k$ ), due to their connection with mutually orthogonal latin squares (MOLS). After adopting some notations and results of Füredi and Gyárfás, we prove the spectral analog of Füredi and Gyárfás's result and obtain the following extremal spectral result. Theorem 5 also implies that the maximum spectral radius of the adjacency tensor of hypergraphs is closely related to the Turán numbers of hypergraphs.

Theorem 5. Let $\mathcal{H}$ denote the set of linear $k$-uniform hypergraphs of order $n, n \equiv 0$ $(\bmod k)$, with forbidden $\mathrm{Fan}^{k}$ and $\rho$ be the maximum spectral radius of hypergraphs in $\mathcal{H}$. For $n$ sufficiently large, we have $\rho=\frac{n}{k}$.

Proof. Let $H=(V, E)$ be a $k$-uniform Fan $^{k}$-free linear hypergraph on $n$ vertices. Let $u$ be the vertex with maximum eigenvector entry. Set $B_{u}=V \backslash N_{u}$, where $N_{u}$ is the neighborhood of the vertex $u$. Suppose $f \in E_{k}\left(N_{u}\right)$, the vertices $\left\{v_{i}\right\}_{i=1}^{k}$ of $f$ must belong to $k$ distinct edges $\left\{e_{i}\right\}_{i=1}^{k}$ containing $u$ since $H$ is linear. But then, $\left\{f, e_{1}, \ldots, e_{k}\right\}$ is the edge set of a $\mathrm{Fan}^{k}$, a contradiction. In other words, $e_{k}\left(N_{u}\right)=0$. On one hand, by Corollary 3, we have

$$
\begin{aligned}
\rho^{2} & \leqslant \frac{1}{k-1}\left[e_{1}\left(N_{u}\right)+2 e_{2}\left(N_{u}\right)+\cdots+(k-1) e_{k-1}\left(N_{u}\right)\right] \\
& \leqslant \frac{1}{k-1}\left[(k-1) e_{1}\left(N_{u}\right)+(k-1) e_{2}\left(N_{u}\right)+\cdots+(k-1) e_{k-1}\left(N_{u}\right)\right] \\
& \leqslant e_{1}\left(N_{u}\right)+e_{2}\left(N_{u}\right)+\cdots+e_{k-1}\left(N_{u}\right) \\
& =e\left(N_{u}, B_{u}\right)
\end{aligned}
$$

By Theorem 4, we know that $|E(H)| \leqslant \frac{n^{2}}{k^{2}}$. Thus $e\left(N_{u}, B_{u}\right) \leqslant \frac{n^{2}}{k^{2}}$. After using (12), we have $\rho^{2} \leqslant \frac{n^{2}}{k^{2}}$, i.e., $\rho \leqslant \frac{n}{k}$.

On the other hand, because a transversal design $T(n, k)$ is an $\frac{n}{k}$-regular linear hypergraph without $\operatorname{Fan}^{k}$, then $\rho(T(n, k))=\frac{n}{k}$. Since $\rho$ is the maximum spectral radius of hypergraphs in $\mathcal{H}$, we have $\rho \geqslant \frac{n}{k}$.

This completes the proof.

\subsection{Spectral radius of linear hypergraphs without Berge $C_{4}$}

One of the first results concerning Turán numbers of Berge cycles is due to Lazebnik and Verstraëte [19]. Very recently this was strengthened by Ergemlidze, Győri and Methuku [8] who showed that $e x_{3}^{\operatorname{lin}}\left(n,\left\{C_{4}\right\}\right) \leqslant \frac{1}{6} n^{\frac{3}{2}}+O(n)$ (which is tight, due to a construction from [19]). Gerbner, Methuku and Vizer [15] proved that $e x_{k}^{\operatorname{lin}}\left(n,\left\{K_{2, t}\right\}\right) \leqslant \frac{\sqrt{t-1}}{k(k-1)} n^{\frac{3}{2}}+O(n)$ which indicates $e x_{k}^{\operatorname{lin}}\left(n,\left\{C_{4}\right\}\right) \leqslant \frac{1}{k(k-1)} n^{\frac{3}{2}}+O(n)$. In this subsection, we give a spectral version of Ergemlidze, Győri and Methuku's results on $k$-uniform linear hypergraphs. Throughout this subsection, suppose $k \geqslant 3$. 
As before, let $H$ be a $k$-uniform linear hypergraph of order $n$ and size $m$ containing no Berge $C_{4}$. Our plan is to first upper bound $\sum_{v \in N_{u}} d(v)$ for the vertex $u$ in $H$. After that, by Corollary 3, we get an upper bound of the spectral radius of a hypergraph containing no Berge $C_{4}$.

Theorem 6. Let $\mathcal{H}$ denote the set of linear $k$-uniform hypergraphs of order $n$ with forbidden Berge $C_{4}$ and $\rho$ be the maximum spectral radius among hypergraphs in $\mathcal{H}$. For $k \geqslant 3$, $\rho \leqslant \sqrt{\frac{3}{2}+\frac{1}{2(k-1)}}(n-1)^{\frac{1}{2}}+O\left(\frac{1}{\sqrt{n}}\right)$.

To prove Theorem 6 , we only need to upper bound $\sum_{v \in N_{u}} d(v)$ by Corollary 3. Notice that $d(v)=\sum_{t=1}^{k} e_{t}^{v}\left(N_{u}\right)$ for any $v \in N_{u}$. Our plan is to estimate via an upper bound on $e_{t}^{v}\left(N_{u}\right)$ for $1 \leqslant t \leqslant k$.

Set $B_{u}=V \backslash\left(N_{u} \cup\{u\}\right)$. Define the set $S_{x}=\left\{\omega \in B_{u} \mid \exists l \in E_{1}^{x}\left(N_{u}\right)\right.$ with $\left.\omega \in l\right\}$ for any $x \in N_{u}$, i.e., the set of vertices contained in some edge which intersects $N_{u}$ only at $x$. Note that $S_{x} \subset N_{x} \backslash\left(N_{u} \cup\{u\}\right)$. The following results are necessary to our proof.

Lemma 7. Let $x$ and $y$ be two adjacent vertices in $H$. Then $\left|N_{x y}\right| \leqslant 2 k-3$.

Proof. Since $x$ and $y$ are adjacent, let $l_{x y}$ be the edge containing both the vertices $x$ and $y$. Suppose for the sake of a contradiction that $\left|N_{x y}\right| \geqslant 2 k-2$. Then there are at least $k$ vertices in $N_{x y}$ other than the common neighbors in $l_{x y}$. Since $H$ is $k$-uniform, there must exist two distinct vertices $v_{1}$ and $v_{2}$ in $N_{x y}$ such that the pairs $x v_{1}$ and $x v_{2}$ are contained in two distinct edges which are not the edge $l_{x y}$.

If the pairs $y v_{1}$ and $y v_{2}$ are contained in one edge incident to $y$, there must exist a vertex $v_{3}$ in $N_{x y}$ such that $y v_{1}$ and $y v_{3}$ are contained in two distinct edges incident to $y$. Then either the edges containing $x v_{1}, y v_{1}, y v_{3}$ and $x v_{3}$ or the edges containing $x v_{2}, y v_{2}$, $y v_{3}$ and $x v_{3}$ form a Berge $C_{4}$ in $H$, a contradiction. Otherwise the pairs $y v_{1}$ and $y v_{2}$ are contained in two different edges incident to $y$, then the four edges containing the pairs $x v_{1}, x v_{2}, y v_{1}$ and $y v_{2}$ form a Berge $C_{4}$ in $H$, a contradiction.

Lemma 8. Let $x$ and $y$ be two distinct vertices in $N_{u}$ of $H$. If the pairs $x u$ and $y u$ are in distinct edges, then $S_{x} \cap S_{y}=\varnothing$.

Proof. Let $l_{x}, l_{y}$ be two edges incident to $u$ such that $x \in l_{x}$ and $y \in l_{y}$. Since $H$ is linear, it is obvious that $l_{x} \neq l_{y}$. If there were a vertex $v$ in $S_{x} \cap S_{y}$, then each of the pairs $x v$ and $y v$ would be contained in edges $l_{x}^{\prime}$ and $l_{y}^{\prime}$ so that $l_{x}^{\prime} \cap N_{u}=\{x\}$ and $l_{y}^{\prime} \cap N_{u}=\{y\}$, whence $l_{x}^{\prime} \neq l_{y}^{\prime}$ and neither edge contains $u$. But then the four edges $l_{x}, l_{y}, l_{x}^{\prime}$, and $l_{y}^{\prime}$ are a Berge $C_{4}$, a contradiction.

Proof of Theorem 6. First we show that $\sum_{t=2}^{k} e_{t}^{v}\left(N_{u}\right)$ is no more than $k$ for any $v \in N_{u}$ while $k \geqslant 3$. Suppose for a contradiction that there is a vertex $v \in N_{u}$ such that $\sum_{t=2}^{k} e_{t}^{v}\left(N_{u}\right) \geqslant k+1$. Since $H$ is linear, there is exactly one of these at least $k+1$ edges containing $u$. Let $l_{i}, 1 \leqslant i \leqslant k$, denote $k$ other edges containing $v$. Since $\left|l_{i} \cap N_{u}\right| \geqslant 2$, we 
select $k$ distinct vertices $x_{i} \in l_{i} \cap N_{u}$ for $1 \leqslant i \leqslant k$. Then for the adjacent vertices $u$ and $v$ we have $\left|N_{u v}\right| \geqslant 2 k-2$, contradicting with Lemma 7 . Thus $d(v) \leqslant e_{1}^{v}\left(N_{u}\right)+k$ for any $v \in N_{u}$.

Since $H$ is linear and $e_{1}^{v}\left(N_{u}\right) \geqslant d(v)-k$ for any $v \in N_{u}$, we have $\left|N_{u}\right|=(k-1) d(u)$ and $\left|S_{v}\right| \geqslant(k-1)(d(v)-k)$. Thus,

$$
\sum_{v \in N_{u}}\left|S_{v}\right| \geqslant \sum_{v \in N_{u}}(k-1)(d(v)-k)=\sum_{v \in N_{u}}(k-1) d(v)-k(k-1)^{2} d(u) .
$$

For each $1 \leqslant i \leqslant d(u)$, let $l_{i}$ denote the $i$-th edge incident to $u$, and write $l_{i}=$ $\left\{u, i_{2}, i_{3}, \cdots, i_{k}\right\}$. Set $V_{i}=\bigcup_{j=2}^{k} S_{i_{j}}$ for each $1 \leqslant i \leqslant d(u)$. Obviously $\bigcup_{i=1}^{d(u)} V_{i} \subseteq B_{u}$.

By Lemma 8, we have $V_{i} \cap V_{j}=\varnothing$ since $l_{i} \cap l_{j}=\{u\}$ by linearity. Thus $\sum_{i=1}^{d(u)}\left|V_{i}\right| \leqslant\left|B_{u}\right|$. For a fixed edge $l_{i}$, we only need to estimate the upper bound of $\left|S_{i_{p}} \cap S_{i_{q}}\right|$ where $i_{p}, i_{q} \in l_{i}$.

Note that

$$
\left|V_{i}\right|=\left|\bigcup_{j=2}^{k} S_{i_{j}}\right| \geqslant \sum_{j=2}^{k}\left|S_{i_{j}}\right|-\sum_{2 \leqslant p<q \leqslant k}\left|S_{i_{p}} \cap S_{i_{q}}\right| .
$$

By Lemma 7, we have

$$
\left|S_{i_{p}} \cap S_{i_{q}}\right| \leqslant\left|N_{i_{p} i_{q}} \backslash\left(N_{u} \cup\{u\}\right)\right| \leqslant\left|N_{i_{p} i_{q}} \backslash\left(l_{i} \backslash\{x, y\}\right)\right| \leqslant(2 k-3)-(k-2)=k-1
$$

since the vertices $p$ and $q$ are adjacent. Then

$$
\left|V_{i}\right| \geqslant \sum_{j=2}^{k}\left|S_{i_{j}}\right|-\left(\begin{array}{c}
k-1 \\
2
\end{array}\right)(k-1)=\sum_{j=2}^{k}\left|S_{i_{j}}\right|-\frac{(k-1)^{2}(k-2)}{2} .
$$

Thus we have

$$
\sum_{j=2}^{k}\left|S_{i_{j}}\right| \leqslant\left|V_{i}\right|+\frac{(k-1)^{2}(k-2)}{2}
$$

and

$$
\sum_{i=1}^{d(u)} \sum_{j=2}^{k}\left|S_{i_{j}}\right| \leqslant \sum_{i=1}^{d(u)}\left|V_{i}\right|+\sum_{i=1}^{d(u)} \frac{(k-1)^{2}(k-2)}{2} .
$$

Since $\sum_{i=1}^{d(u)}\left|V_{i}\right| \leqslant\left|B_{u}\right| \leqslant n$, applying (15), we obtain

$$
\begin{aligned}
\sum_{i=1}^{d(u)} \sum_{j=2}^{k}\left|S_{i_{j}}\right| & \leqslant \sum_{i=1}^{d(u)}\left|V_{i}\right|+\sum_{i=1}^{d(u)} \frac{(k-1)^{2}(k-2)}{2} \\
& \leqslant\left|B_{u}\right|+\frac{(k-1)^{2}(k-2)}{2} d(u)
\end{aligned}
$$




$$
\leqslant n-\left|N_{u}\right|+\frac{(k-1)^{2}(k-2)}{2} d(u) \text {. }
$$

Combining this with (13), then

$$
\sum_{v \in N_{u}}(k-1)(d(v)-k) \leqslant \sum_{i=1}^{d(u)} \sum_{j=2}^{k}\left|S_{i_{j}}\right| \leqslant n+\frac{(k-1)^{2}(k-2)}{2} d(u)-(k-1) d(u) .
$$

The first inequality follows $\left|S_{v}\right| \geqslant(k-1)(d(v)-k)$ for any $v \in N_{u}$ and the second because $\left|N_{u}\right|=(k-1) d(u)$. Simplifying (17), we have

$$
\sum_{v \in N_{u}}(k-1) d(v) \leqslant n+\frac{k(k-1)(3 k-5)}{2} d(u) .
$$

Since $H$ is linear, it is obvious that $d(u) \leqslant \frac{n-1}{k-1}$. Combining (18) with Corollary 3, we get

$$
\rho^{2} \leqslant \frac{n}{(k-1)^{2}}+\frac{k(3 k-5)}{2(k-1)} d(u) \leqslant\left(\frac{3}{2}+\frac{1}{2(k-1)}\right)(n-1)+\frac{1}{(k-1)^{2}}
$$

That is, $\rho \leqslant \sqrt{\frac{3}{2}+\frac{1}{2(k-1)}}(n-1)^{\frac{1}{2}}+O\left(\frac{1}{\sqrt{n}}\right)$. This completes the proof.

Note that $\sum_{t=2}^{k} e_{t}^{v}\left(N_{u}\right) \leqslant k$ holds if $k \geqslant 3$ in the proof of Theorem 6 . For a graph $G$ of order $n$ containing no $C_{4}$, we have $e_{2}^{v}\left(N_{u}\right) \leqslant 1$ and $\rho(G) \leqslant \sqrt{n}$ after applying $e_{2}^{v}\left(N_{u}\right) \leqslant 1$ to the proof of Theorem 6. Recall that Nikiforov [24] proved that $\rho^{2}-\rho \leqslant n-1$ where $\rho$ is the spectral radius of a graph $G$ of order $n$ containing no $C_{4}$. Obviously, $\rho(G) \leqslant \sqrt{n}$ is asymptotically equivalent to the Nikiforov's result when $n$ is sufficiently large.

\subsection{Spectral radius of linear hypergraphs with at least girth five}

The question we consider in this subsection is to determine the maximum spectral radius of $k$-uniform linear hypergraphs on $n$ vertices of girth at least five, while the maximum number of edges in graphs of girth five is an old problem of Erdős [7]. Lazebnik and Verstraëte [19] showed that $e x_{3}^{\operatorname{lin}}\left(n,\left\{C_{3}, C_{4}\right\}\right)=n^{3 / 2} / 6+O(n)$. Very recently this was strengthened by Ergemlidze, Győri, and Methuku, who showed in [8] that $\operatorname{ex}_{3}^{\operatorname{lin}}\left(n,\left\{C_{3}, C_{4}\right\}\right) \sim$ $e x_{3}^{\operatorname{lin}}\left(n,\left\{C_{4}\right\}\right)$. Timmons [28] proved that $e x_{k}^{\operatorname{lin}}\left(n,\left\{C_{3}, K_{2, t+1}\right\}\right) \leqslant \frac{\sqrt{t}}{k(k-1)} n^{3 / 2}+O(n)$ which indicated $e x_{k}^{\operatorname{lin}}\left(n,\left\{C_{3}, C_{4}\right\}\right) \leqslant \frac{1}{k(k-1)} n^{3 / 2}+O(n)$. Now we investigated on the spectral version of $k$-uniform linear hypergraphs on $n$ vertices of girth at least five while the proof of the result below is substantially simpler and shorter than the above proof.

Theorem 9. Let $\mathcal{H}$ denote the set of linear $k$-uniform hypergraphs of order $n$ with girth at least five and $\rho$ be the maximum spectral radius of hypergraphs in $\mathcal{H}$. For $n$ sufficiently large, we have $\rho \leqslant \sqrt{\frac{n-1}{k-1}}+O\left(\frac{1}{\sqrt{n}}\right)$. 
Proof. Let $H$ be a linear hypergraphs of order $n$ and size $m$ with girth at least five. For an arbitrary and fixed vertex $u$, it is easy to verify that $E_{k-1}^{v}\left(N_{u}\right)$ is exactly the set of edges incident to $u$ and $E_{t}^{v}\left(N_{u}\right)=\varnothing$ if $t \notin\{1, k-1\}$, where $v$ is an arbitrary vertex in $N_{u}$.

Otherwise $H$ contains a Berge $C_{3}$, a contradiction. That is to say $\sum_{t=2}^{k} t e_{t}\left(N_{u}\right)=(k-1) d(u)$. Now we only need to provide an upper bound on $e_{1}\left(N_{u}\right)$.

As before, set $B_{u}=V \backslash\left\{N_{u} \cup\{u\}\right\}$ and $S_{v}=\left\{\omega \in B_{u} \mid \exists l \in E_{1}^{v}\left(N_{u}\right)\right.$ with $\left.\omega \in l\right\}$ for any $v \in N_{u}$. By Lemma 8 , it is easy to verify that $S_{x} \cap S_{y}=\varnothing$ for an arbitrary pair $x, y$ in $N_{u}$ if $x$ and $y$ are nonadjacent. If $x$ and $y$ are adjacent, suppose for a contradiction that there is a vertex $z \in S_{x} \cap S_{y}$. Let $l_{x y}$ be the edge containing both the vertices $x$ and $y$. Similarly for $l_{x z}$ and $l_{y z}$. Since $H$ is linear, it is clear that $l_{x y}, l_{x z}$ and $l_{y z}$ form a Berge $C_{3}$, a contradiction. Thus $e_{1}\left(N_{u}\right) \leqslant(n-(k-1) d(u)) /(k-1)$.

By Corollary 3, we get

$$
\rho^{2} \leqslant \frac{1}{k-1}\left[e_{1}\left(N_{u}\right)+2 e_{2}\left(N_{u}\right)+\cdots+k e_{k}\left(N_{u}\right)\right] \leqslant \frac{n}{(k-1)^{2}}+\frac{(k-2) d(u)}{k-1} .
$$

Since $H$ is linear, it is obvious that $d(u) \leqslant \frac{n-1}{k-1}$, we get

$$
\rho^{2} \leqslant \frac{n-1}{k-1}+\frac{1}{(k-1)^{2}} .
$$

Therefore $\rho \leqslant \sqrt{\frac{n-1}{k-1}}+O\left(\frac{1}{\sqrt{n}}\right)$.

If $G$ be a graph of order $n$ containing neither $C_{3}$ nor $C_{4}$, it is easy to see that for $n$ sufficiently large, Theorem 9 is asymptotically equivalent to the result of Favaron, Mahéo and Saclé [9] who showed that $\rho(G) \leqslant \sqrt{n-1}$ where $\rho(G)$ is the spectral radius of $G$.

\section{Conclusion and open problems}

Of course, the above results are just a small sample of spectral analogues for Turántype hypergraph problems. Spectral extremal problems can be a rich font of interesting questions, since so many classical extremal problems have been investigated. See [17] for much more along these lines. Here we outline a few related to the above questions that we find particularly appealing.

1. What do the extremal spectral results for other hereditary linear hypergraph classes look like? For example, one might consider forbidden Berge cycles with lengths greater than 4 , Berge $K_{2, t}$, the Fano plane, etc.

2. To get the upper bounds of Theorem 6 and Theorem 9, we used the inequality $d(u) \leqslant \frac{n-1}{k-1}$ which indicates that equality holds if there exists a vertex adjacent to other vertices in $H$. So how to construct the hypergraph with spectral radius attaining $O(\sqrt{n})$ ? As we know, the hyperstar containing either $C_{3}$ nor $C_{4}$ with $\frac{n-1}{k-1}$ edges sharing one vertex in common, can't reach the upper bound in Theorem 9 , which is different from the case for graphs. 
3. How would one generalize Lemma 1 to other tensors, such as the (signless) Laplacian tensor? How large can the maximum eigenvalue of the (signless) Laplacian tensor of a uniform hypergraph be if various subhypergraphs are forbidden?

4. Kalai asked the following beautiful question: Let $\mathcal{F}_{n}$ denote the family of linear 3-uniform hypergraphs $H=(V, E)$ for which there exists an injective function $\phi: V \rightarrow \mathbb{R}^{3}$ so that the convex hulls of $\phi(e)$ and $\phi(f)$ intersect exactly in $\phi(e \cap f)$ for every $e, f \in E$. In other words, $\mathcal{F}_{n}$ consists of those linear 3-uniform hypergraphs whose edges can be faithfully embedded as planar triangles in $\mathbb{R}^{3}$. Then, if $f(n)$ is the maximum number of edges of any hypergraph in $\mathcal{F}_{n}$, is it true that $f(n)=o\left(n^{2}\right)$ ? Károlyi and Solymosi showed in [16] that $f(n)=\Omega\left(n^{3 / 2}\right)$, but not much more is known about this question. We ask the spectral analogue: for $H \in \mathcal{F}_{n}$, is it true that $\rho(H)=o(n)$ ?

\section{References}

[1] S. Bai, L. Lu, A bound on the spectral radius of hypergraphs with $e$ edges, Linear Algebra Appl., 549(2018) 203-218.

[2] C. Berge, Hypergraph: Combinatorics of Finite Sets, third edition, North-Holland, Amsterdam, 1973.

[3] L. Babai, B. Guiduli, Spectral extrema for graphs: the Zarankiewicz problem, Electron. J. Combin., 16(2009), \#R123.

[4] K. Chang, K. Pearson, T. Zhang, Perron-Frobenius theorem for nonnegative tensors, Commun. Math. Sci., 6(2008) 507-520.

[5] C. Collier-Cartaino, N. Graber, T. Jiang, Linear Turán numbers of $r$-uniform linear cycles and related Ramsey numbers, Combinatorics, Probability and Computing, 27(2018) 358-386.

[6] J. Cooper, A. Dutle, Spectra of uniform hypergraphs, Linear Algebra Appl., 436 (2012) 3268-3292.

[7] P. Erdős, Some recent progress on extremal problems in graph theory, Congr. Numer., 14(1975) 3-14.

[8] B. Ergemlidze, E. Győri, A. Methuku, Asymptotics for Turán numbers of cycles in 3-uniform linear hypergraphs, J. Comb. Theory, Ser. A, 163(2019) 163-181.

[9] O. Favaron, M. Mahéo, J.-F. Saclé, Some eigenvalue properties in graphs(conjectures of Graffiti.II), Discrete Math., 111(1993) 197-220.

[10] Z. Füredi, L. Özkahya, On 3-uniform hypergraphs without a cycle of a given length, Discrete Appl. Math., 216(2017) 582-588.

[11] Z. Füredi, M. Simonovits, The history of degenerate (bipartite) extremal graph problems, In Erdős Centennial (pp. 169-264), Springer Berlin Heidelberg 2013.

[12] Z. Füredi, A. Gyárfás, An Extension of Mantel's Theorem to k-Graphs, Am. Math. Mon., 127(2020) 263-268. 
[13] S. Friedland, A. Gaubert, L. Han, Perron-Frobenius theorems for nonnegative multilinear forms and extensions, Linear Algebra Appl., 438(2013) 738-749.

[14] D. Gerbner, C. Palmer, Extremal results for Berge-hypergraphs, SIAM J. Discrete Math., 31(2017) 2314-2327.

[15] D. Gerbner, A. Methuku, M. Vizer, Asymptotics for the Turán number of Berge $K_{2, t}$, J. Comb. Theory, Ser. B, 137(2019) 264-290.

[16] G. Károlyi, J. Solymosi, Almost disjoint triangles in 3-space. Disc. Comput. Geom., 28(2002), no. 4, 577-583.

[17] P. Keevash, J. Lenz, D. Mubayi, Spectral extremal problems for hypergraphs, SIAM J. Discrete Math., 28(2014), no. 4, 1838-1854.

[18] P. Keevash, Hypergraph Turán problems, Surveys in Combinatorics, 392(2011) 83140.

[19] F. Lazebnik, J. Verstraëte, On hypergraphs of girth five, Electron. J. Combin., 10 (2003) \#R25.

[20] L. Lim, Singular values and eigenvalues of tensors: a variational approach, in: Proceedings of the IEEE International Workshop on Computational Advances in MultiSensor Adaptive Processing (CAMSAP 05), 1(2005) 129-132.

[21] D. Mubayi, O. Pikhurko, A new generalization of Mantel's theorem to $k$-graphs, J. Comb. Theory Series B, 97(2007) 669-678.

[22] D. Mubayi, J. Verstraëte, A survey of Turán problems for expansions, In Recent Trends in Combinatorics, Springer International Publishing, (2016) 117-143.

[23] V. Nikiforov, Some new results in extremal graph theory, in: Surveys in Combinatorics, London Math. Soc. Lecture Notes, 392(2011) 141-181.

[24] V. Nikiforov, Bounds on graph eigenvalues II, Linear Algebra Appl., 427(2007) 183189.

[25] L. Qi, Eigenvalues of a real supersymmetric tensor, J. Symb. Comput., 40(2005) 1302-1324.

[26] I. Ruzsa, E. Szemerédi, Triple systems with no six points carrying three triangles, Combinatorica, 18(1976) 939-945.

[27] R. Stanley, A bound on the spectral radius of graphs with e edges, Linear Algebra Appl., 87(1987) 267-269.

[28] C. Timmons, On $r$-uniform linear hypergraphs with no Berge- $K_{2, t}$, Electron. J. Combin., 24(4) (2017) \#P4.34.

[29] H. Wilf., Spectral bounds for the clique and independence numbers of graphs, J. Comb. Theory, Ser. B, 40(1986) 113-117.

[30] Y. Yang, Q. Yang, Further results for Perron-Frobenius theorem for nonnegative tensors, SIAM J. Matrix Anal. Appl., 31(2010) 2517-2530. 